\title{
Hybrid Laser-Plasma Micro-Structuring of Fused Silica Based on Surface Reduction by a Low-Temperature Atmospheric Pressure Plasma
}

\author{
Stephan BRÜCKNER ${ }^{*}$, Jennifer HOFFMEISTER ${ }^{* *}$, Jürgen IHLEMANN ${ }^{* * *}$, Christoph GERHARD ${ }^{*}$, Stephan WIENEKE ${ }^{* *}$ \\ and Wolfgang VIÖL ${ }^{* *}$
}

\author{
${ }^{*}$ Clausthal University of Technology, Institute of Energy Research and Physical Technologies, Leib- \\ nizstraße 4, 38678 Clausthal-Zellerfeld, Germany \\ ${ }^{* *} H A W K$ - University of Applied Sciences and Arts, Laboratory of Laser and Plasma Technologies, \\ Von-Ossietzky-Straße 99, 37085 Göttingen, Germany \\ ${ }^{* * *}$ Laser Laboratory Göttingen e.V., Hans-Adolf-Krebs-Weg 1, 37077 Göttingen, Germany \\ E-mail: vioel@hawk-hhg.de
}

\begin{abstract}
In this contribution, we report on a novel hybrid laser-plasma method for material processing applications. This method is based on the combination of both an ArF excimer laser $(\lambda=193 \mathrm{~nm})$ and a low-temperature atmospheric pressure plasma jet source for the chemical reduction of glass surfaces. Here, a hydrogen-containing plasma gas was applied. Due to the layer of silicon suboxide that is generated in this vein, the absorption of the incoming machining laser beam is significantly increased after 15 minutes of plasma-treatment. Several machining experiments in terms of frontside ablation were performed on fused silica. Here, both pure and plasma-treated surfaces were ablated using single laser pulses with a pulse duration of $20 \mathrm{~ns}$. By introducing the presented hybrid technique, the ablation threshold for micro-structuring was reduced significantly by a factor of 4.6 whereas the peak-to-valley height $R z$ of the machined area was decreased by a factor of 2.3. Further, back-side ablation using the presented method was considered. By a terminal tempering process, the initial transmission characteristics of fused silica can be reconstituted.

DOI:10.2961/jlmn.2012.01.0014
\end{abstract}

Keywords: hybrid laser-plasma technology, atmospheric pressure plasma, fused silica, silicon suboxide, micro-structuring

\section{Introduction}

Due to its specific properties, fused silica is a wellestablished and suitable optical medium for the production of a variety of optical components such as UV-transparent optics, semiconductor devices and integrated micro-optical elements. Regarding laser based methods for the manufacture of such components, material removal of fused silica and glasses in general can be achieved by several techniques such as laser ablation using UV-, IR- or NIR-laser radiation [1-3], or laser induced backside wet etching [4,5]. Further, laser induced etching techniques introducing UVabsorbing films such as toluene [6] and carbon [7] or laser induced plasma-assisted ablation [8] can be applied. Beyond, an indirect processing method of fused silica surfaces consists of the vacuum deposition and laser structuring of UV-absorbing silicon suboxide layers $\left(\mathrm{SiO}_{\mathrm{X}}\right)$ and their subsequent oxidation to $\mathrm{SiO}_{2}$ [9]. Also, since fused silica consists of pure silicon dioxide $\left(\mathrm{SiO}_{2}\right)$, its surface can be directly reduced to silicon suboxide $\left(\mathrm{SiO}_{\mathrm{X}}\right.$, where $\left.1<\mathrm{X}<2\right)$ or silicon monoxide $(\mathrm{SiO})$ by applying hydrogenous gases at high temperatures [10].

The presented hybrid laser-plasma removal method is based on the surface reduction of fused silica by a lowtemperature atmospheric pressure plasma and a subsequent laser ablation. To our best knowledge, this is the first work on surface processing of fused silica using such a plasma source. In contrast to the pulsed laser deposition method (PLD) [11] or the deposition of metastable silicon suboxide by vacuum evaporation [9], the introduced plasma gener- ates atomic hydrogen from the used forming gas which directly generates a suboxidal layer onto the fused silica substrate. This effect allows a significant decrease in required energy for laser ablation of fused silica.

\section{Experimental setup and experimentation}

For the plasma-treatment of the investigated $2 \mathrm{~mm}$ thick fused silica samples, a low-temperature potential-free atmospheric pressure plasma jet "kinpen 09" from neoplas tools $\mathrm{GmbH}$ was applied. The plasma source was directed perpendicular onto the sample's surface. During the plasma-treatment, the samples were moved by a xy-linear stage. The working distance of the plasma jet nozzle to the fused silica surface was $1 \mathrm{~mm}$. In order to initialise the reduction process, forming gas $90 / 10$ (consisting of $90 \%$ nitrogen and $10 \%$ hydrogen) was used as working gas. The treatment time at each point of the sample surface was varied in the range from 0 to 15 minutes with a gas flow rate of $25 \mathrm{slm}$. After 5 and 15 minutes, transmission spectra (including reflexion losses) were taken in order to verify the reduction progress with respect to a pure fused silica sample.

The subsequent laser ablation was performed after a maximum plasma-treatment duration of 15 minutes using an ArF excimer laser "LPX 315" from Lambda Physik $(\lambda=193 \mathrm{~nm})$. By an optical setup, consisting of two convex lenses $\left(f_{1}=750 \mathrm{~mm}, f_{2}=100 \mathrm{~mm}\right)$, a mask imaging was realised in order to image a diaphragm on the fused silica sample's surface as shown in figure 1 . 


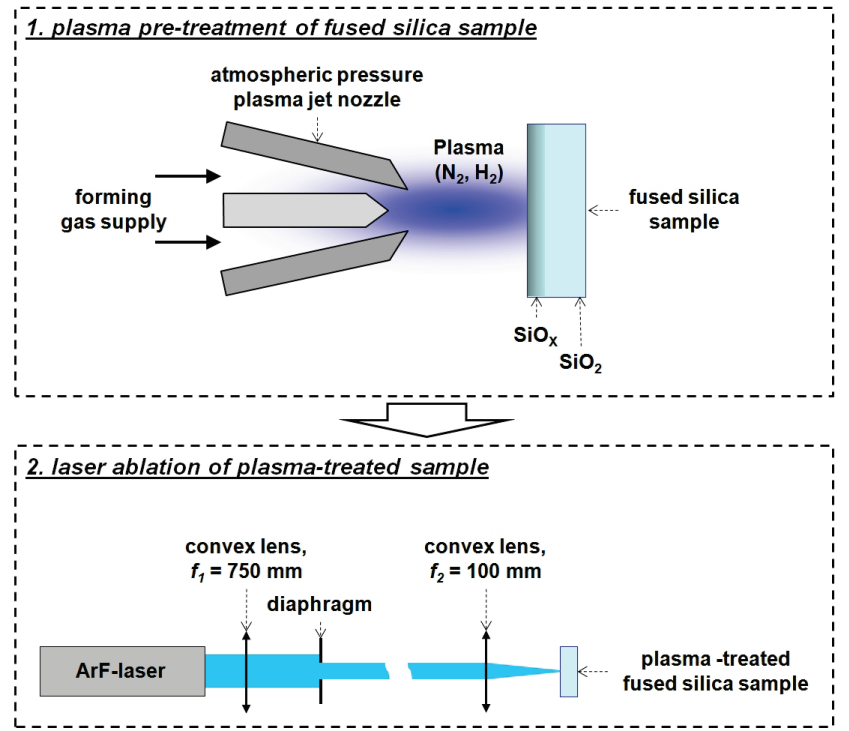

Fig. 1 Schematic and functional principle of the setup for plasma pre-treatment and subsequent front-side ablation of fused silica.

The aperture of this diaphragm was $3 \mathrm{~mm}$. The demagnification was about 15-times, leading to an irradiated spot of about $200 \mu \mathrm{m}$ in diameter on the front-side of the sample. In addition to this standard front-side configuration, some experiments in the back-side configuration (where the beam passes through the sample and the image plane is on the back-side [1]) have been carried out.

In order to investigate the influence of the plasmatreatment on the machining properties of the fused silica surface, the fluence threshold $\Phi_{\min }$ for substrate ablation of both treated and untreated reference surfaces was determined by applying a series of single laser pulses with increasing energy. The pulse duration $t$ was 20 ns. Following, ablation experiments were performed just above the particular ablation threshold.

\section{Results and discussion}

By applying the plasma-treatment, the transmission of the investigated fused silica samples was reduced significantly as shown in figure 2 .

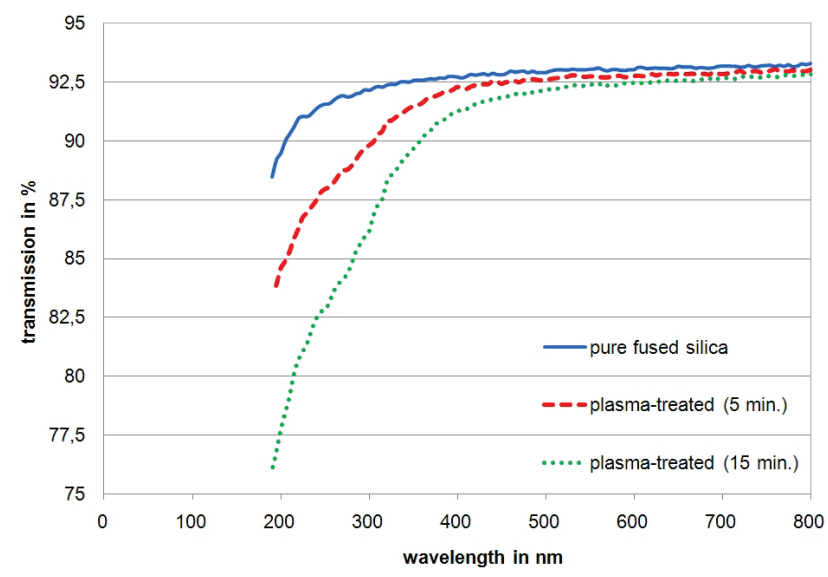

Fig. 2 Transmission spectra of a pure fused silica sample (solid line) and after plasma-treatment for $5 \mathrm{~min}$ (dashed line) and 15 min (dotted line).
In comparison to a pure fused silica sample, the transmission at the wavelength of the used laser of $193 \mathrm{~nm}$ was reduced by a total percental value of $5.8 \%$ after 5 minutes and $14.1 \%$ after 15 minutes plasma-treatment.

For front-side ablation of pure fused silica using a single laser pulse with a pulse duration of $t=20 \mathrm{~ns}$, an ablation threshold of $6 \mathrm{~J} / \mathrm{cm}^{2}$ was determined. In comparison, the plasma-treated surfaces show a significant decrease in required fluence for ablation due to the increased absorbance by means of the reducing-acting forming gas. Here, front-side ablation was already achieved at a fluence of $1.3 \mathrm{~J} / \mathrm{cm}^{2}$. Hence, the ablation threshold was reduced by a factor of 4.6 by applying the plasma-treatment. Furthermore, disturbing effects such as micro-cracks and melt are avoided in this vein. The resulting geometry was measured using a confocal scanning microscope "PL $\mathrm{P} 2300$ " from Sensofar as shown in figure 3.
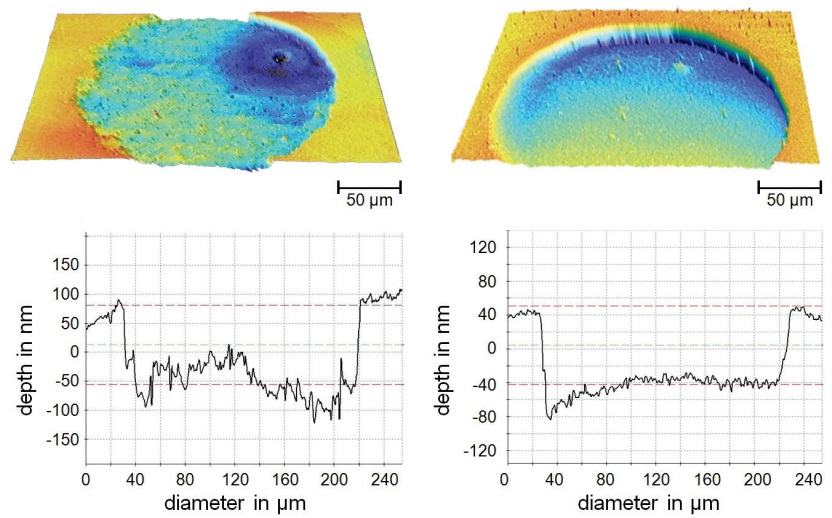

Fig. 3 Isometric projection (above) and cross-section (below) of pure (left) and plasma-treated (right) fused silica surfaces after single pulse front-side ablation near the particular ablation threshold $\left(6 \mathrm{~J} / \mathrm{cm}^{2}\right.$ left, $1.8 \mathrm{~J} / \mathrm{cm}^{2}$ right $)$.

In addition to the above-mentioned reduction of ablation threshold, the peak-to-valley height $R z$ of the plasmatreated machined area $(R z=34.7 \mathrm{~nm})$ is reduced by a factor of 2.3 with respect to pure laser ablated fused silica, where $R z=79.8 \mathrm{~nm}$. As shown in figure 4, in the case of the plasma-treated surface. the complete irradiated spot of about $200 \mu \mathrm{m}$ diameter is ablated with a smooth surface and perfect contour accuracy according to the mask diaphragm. In contrast, for the untreated surface, the ablated area looks quite porous and does not fill the complete irradiated spot.
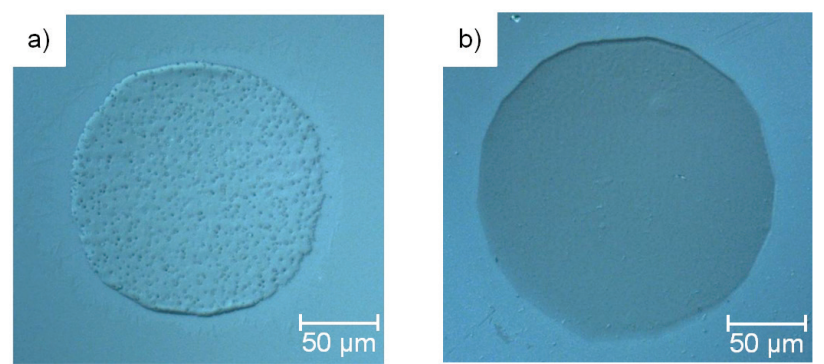

Fig. 4 Microscope image of the pure (a) and plasma-treated (b) fused silica sample after front-side ablation with a single pulse near the particular ablation threshold $\left(6 \mathrm{~J} / \mathrm{cm}^{2}\right.$ left, $1.8 \mathrm{~J} / \mathrm{cm}^{2}$ right $)$. 
In comparison to pure laser ablation, a lower ablation depth is achieved by the presented laser-plasma hybrid method. However, this enables precise control of the ablation profile by fine adjustment of the fluence.

Furthermore, a comparison of both front-side and backside ablation of plasma-treated fused silica surfaces was carried out. Whereas single pulse back-side ablation of untreated samples is not possible with this setup, because ablation starts already at the front-side at the required high fluence, for single-pulse back-side ablation of plasmatreated samples at $1.3 \mathrm{~J} / \mathrm{cm}^{2}$, a higher removal rate was observed. Figure 5 shows both an isometric projection and cross-section of an ablated spot at the back-side of the sample.
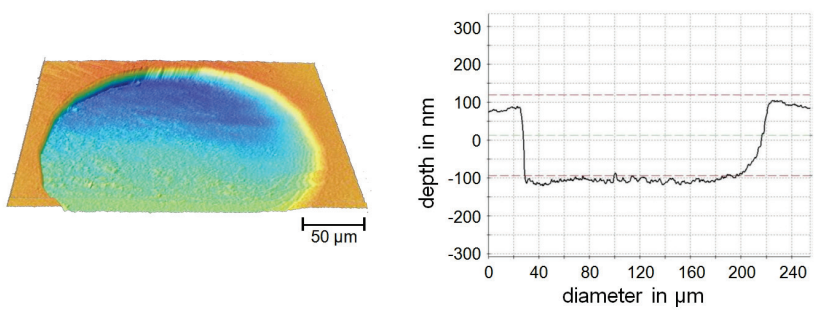

Fig. 5 Isometric projection (left) and cross-section (right) of a plasma-treated fused silica surface after single pulse back-side ablation at a fluence of $1.3 \mathrm{~J} / \mathrm{cm}^{2}$.

Compared to front-side ablation, where the depth of ablation $d$ was $45 \mathrm{~nm}$, the depth of ablation was increased by a factor of 3.9 in the case of single-pulse back-side ablation at a fluence of $1.3 \mathrm{~J} / \mathrm{cm}^{2}(d=175 \mathrm{~nm})$. Comparable increases were also found for higher fluences as shown by the corresponding ablation depths in figure 6 .

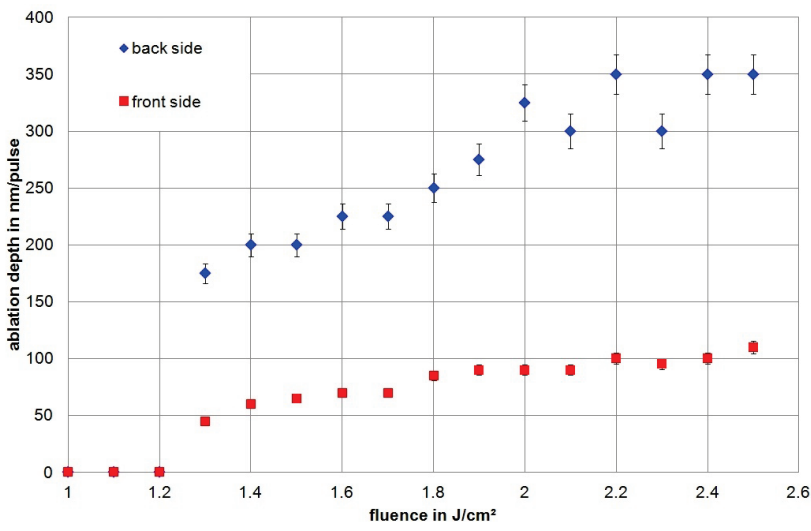

Fig. 6 Ablation depth vs. fluence for both front-side and backside single-pulse ablation of plasma-treated fused silica

In addition, for back-side ablation, the peak-to-valley height $R z$ of $14.5 \mathrm{~nm}$ is 2.4 times lower in comparison to single-pulse front-side ablation at a fluence of $1.3 \mathrm{~J} / \mathrm{cm}^{2}$. Such significant differences in front- and back-side ablation were already observed in previous work and could be explained by the attenuation of the ablating laser pulse by the plume of the removed material, which is only effective for front side irradiation [1]. In the case of ablation at higher number of pulses, the ablation depth increased linearly. When applying 35 laser pulses at $2.4 \mathrm{~J} / \mathrm{cm}^{2}$, an ablation depth of approx. $11 \mu \mathrm{m}$ was achieved, still featuring good machining quality.
After the micro-structuring, the transmission can be increased by a tempering process. For this purpose, the sample was tempered at $1000^{\circ} \mathrm{C}$ in air for inducing a reoxidisation of the plasma-treated layer. As shown in figure 7, the transmission at $\lambda=193 \mathrm{~nm}$ amounts to $83.8 \%$ after 24 hours and $86.8 \%$ after 48 hours of tempering.

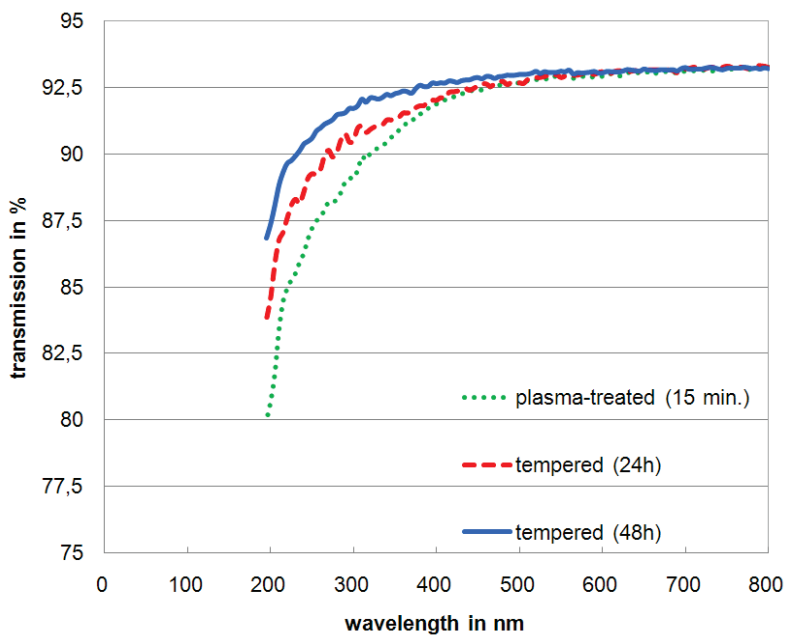

Fig. 7 Transmission spectra of a plasma-treated fused silica sample (dotted line) after 24 hours (dashed line) and 48 hours (solid line) of tempering.

In comparison, a transmission of $88.4 \%$ at $\lambda=193 \mathrm{~nm}$ was measured for the pure, untreated fused silica sample at the beginning. Thus, almost the initial transmission characteristics can be reconstituted in this vein.

\section{Conclusion}

In terms of front-side ablation, the presented hybrid laser-plasma micro-structuring method allows a significant decrease of required fluence for ablation of fused silica by a factor of 4.6. In addition, a significant enhancement of the contour accuracy of the imaged mask was observed. Further, the resulting peak-to-valley height $R z$ of the machined area is reduced by a factor of 2.3. The depth of ablation is reduced by $20 \%$ in the case of laser-plasma singlepulse ablation.

The resulting surface roughness was furthermore reduced by applying back-side ablation. Here, a higher removal rate was obtained additionally.

By a terminal tempering, the plasma-treated samples can be re-oxidised, almost resulting in the initial transmission characteristics. Thus, this technique offers a novel and economic alternative for the manufacture of high-precision micro-structures in fused silica substrates. Further improvement could be achieved by introducing a laser beam homogeniser to the presented optical setup.

\section{Acknowledgments}

The authors gratefully acknowledge the support by the European Regional Development Funds (EFRE) and the Workgroup Innovative Projects of Lower Saxony (AGiP) in the frame of the Lower Saxony Innovation Network for Plasma Technology (NIP). Further, the support by the Federal Ministry of Economics and Technology (BMWi) in the frame of the research project PROKLAMO is gratefully acknowledged. 


\section{References}

[1] J. Ihlemann: Appl. Surf. Sci. 54, (1992) 193-200.

[2] C. Gerhard, F. Druon, P. Blandin, M. Hanna, F. Balembois, P. Georges, F. Falcoz: Appl. Opt., 47/7, (2008) 967-974.

[3] C. Buerhop, B. Blumenthal, R. Weissmann, N. Lutz, S. Biermann: Appl. Surf. Sci. 46, (1990) 430-434.

[4] J. Wang, H. Niino, A. Yabe: Appl. Phys. A 68, (1999) 111-113.

[5] T. Sato, R Kurosaki, Y. Kawaguchi, A. Narazaki, H. Niino: J. Laser. Micro. Nanoen. 5/3, (2010) 256-262

[6] K. Zimmer, R. Böhme, B. Rauschenbach: Appl. Phys. A 79, (2004) 1883-1885.

[7] R. Böhme, K. Zimmer, B. Rauschenbach: Appl. Phys. A 82, (2006) 325-328.

[8] J. Zhang, K. Sugioka, K. Midorikawa: Opt. Let., 23, (1998) 1486-1488.

[9] M. Schulz-Ruhtenberg, J. Ihlemann, J. Heber: Appl. Surf. Sci. 248, (2005) 190-195.

[10] S. T. Tso, J. A. Pask: J. Am. Cer. Soc. 65/9, (1982) 457-460.

[11] J. M. Lackner, W. Waldhauser, R. Ebner, W. Lenz, C. Suess, G. Jakopic, G. Leising, H. Hutter: Surf. Coat. Tech., 163-164, (2003) 300-305.

(Received: November 22, 2011, Accepted: January 4, 2012) 